\title{
Uterine Pain
}

National Cancer Institute

\section{Source}

National Cancer Institute. Uterine Pain. NCI Thesaurus. Code C78694.

A painful sensation in the uterus. 